\title{
A Missense Variant in Granulysin is Associated with the Efficacy of Pegylated-Interferon-Alpha Therapy in Chinese Patients with HBeAg-Positive Chronic Hepatitis B
}

\author{
Jing $\mathrm{Li}^{1}{ }^{1 *}$ \\ Haitao Chen ${ }^{1,2, *}$ \\ Jiaxuan Chen ${ }^{1, *}$ \\ Bin Zhou' \\ Jinlin Hou' \\ De-Ke Jiang'
}

'State Key Laboratory of Organ Failure Research, Guangdong Key Laboratory of Viral Hepatitis Research, Guangdong Institute of Liver Diseases, Department of Infectious Diseases and Hepatology Unit, Nanfang Hospital, Southern Medical University, Guangzhou, People's Republic of China; ${ }^{2} \mathrm{School}$ of Public Health (Shenzhen), Sun Yat-sen University, Shenzhen, People's Republic of China

*These authors contributed equally to this work
Correspondence: De-Ke Jiang; Jinlin Hou State Key Laboratory of Organ Failure Research, Guangdong Key Laboratory of Viral Hepatitis Research, Guangdong Institute of Liver Diseases, Department of Infectious Diseases and Hepatology Unit, Nanfang Hospital, Southern Medical University, Guangzhou, People's Republic of China

Tel +86-20-62786533; +86-20-6I64l94l

Email dekejiangl7@smu.edu.cn;

jlhousmu@I63.com
Purpose: Granulysin (GNLY) is a cytotoxic granule that has been reported to have various antimicrobial activities. We evaluated the association between a missense variant in GNLY (rs11127) and treatment efficacy of pegylated interferon-alpha (PegIFN $\alpha)$ or nucleos(t)ide analogs (NUCs) in patients with chronic hepatitis B (CHB).

Patients and Methods: We included a total of 1823 patients with hepatitis $\mathrm{B}$ e antigen (HBeAg)-positive CHB (954 patients treated with PegIFN $\alpha$ and 869 patients treated with NUCs) in four Phase IV multicenter randomized controlled trials. The association of the GNLY rs11127 genotype with the combined response (CR), defined as HBeAg seroconversion and hepatitis B virus (HBV) DNA level $<2000 \mathrm{IU} / \mathrm{mL}$ was evaluated. A polygenic score (PGS) was constructed to evaluate the cumulative effect of multiple single-nucleotide polymorphisms (SNPs), including rs11127 and several other SNPs, STAT4 rs7574865, CFB rs12614, and CD55 rs28371597, which were reported to be associated with CR.

Results: GNLY rs11127 was significantly associated with $\mathrm{CR}$ in patients treated with PegIFN $\alpha$. The CR rate in patients with the rs $11127 \mathrm{CC}$ genotype was higher than that with the CT or TT genotype $(40.98 \%$ vs $30.34 \%$ or $27.09 \%, P=0.003)$. Furthermore, a PGS integrating GNLY rs11127 and three other SNPs was significantly associated with CR in PegIFN $\alpha$-treated patients $(P<0.001)$. However, no significant correlation was found between GNLY rs11127 and CR in NUCs-treated patients.

Conclusion: GNLY rs11127 is an independent biomarker for predicting the response to PegIFN $\alpha$ therapy in HBeAg-positive CHB patients. Furthermore, the PGS, including GNLY rs11127, provides new insights for individualized treatment in clinical practice.

Keywords: GNLY, rs11127, chronic hepatitis B, PegIFNa treatment, polygenic score

\section{Introduction}

Chronic hepatitis B virus (HBV) infection remains a major concern for global health, with an estimated 292 million cases and approximately $30 \%$ in China. ${ }^{1}$ Because of its high prevalence and severe health consequences, such as cirrhosis and hepatocellular carcinoma (HCC), chronic HBV infection imposes a substantial burden on patients and society. ${ }^{2}$ The current first-line antiviral treatments can be classified into two categories: pegylated interferon-alpha (PegIFN $\alpha$ ) and nucleos(t)ide analogs (NUCs). ${ }^{3}$ NUCs have excellent performance in tolerability, high rates of biochemical remission, and effective inhibition of HBV replication; however, long-term therapies also incur increasing cost, 
safety profiles and compliance issues. As for PegIFN $\alpha$ treatment, despite its severe adverse reactions, it is also an alternative strategy with considerable efficacy in increasing hepatitis B s antigen ( $\mathrm{HBsAg}$ ) loss and reducing the incidence of cirrhosis and HCC. ${ }^{4,5}$ According to the American Association for the Study of Liver Disease (AASLD) 2018 Hepatitis B Guidance, indicators such as HBV DNA suppression, $\mathrm{HBeAg}$ seroconversion or loss, normalization of alanine aminotransferase (ALT), and HBsAg loss, are provided to assess the efficacy of first-line antiviral therapies, including PegIFNa. ${ }^{6}$ Combined response (CR), defined as $\mathrm{HBeAg}$ seroconversion along with HBV DNA level $<2000 \mathrm{IU} / \mathrm{mL}$, was also used to evaluate for IFN efficacy. ${ }^{7,8}$ Regardless of NUCs or PegIFN $\alpha$, the therapeutic response rate is limited. Therefore, it is important to identify novel biomarkers to predict the therapeutic efficacy.

Accumulating evidence has demonstrated that host genetics could affect the response to IFN $\alpha$ treatment. ${ }^{9,10}$ Notably, IL28B polymorphism (rs12979860), a predictor of spontaneous clearance of hepatitis $\mathrm{C}$ virus (HCV), was strongly associated with therapy response in patients with chronic HCV treated with IFN $\alpha$ and ribavirin. ${ }^{11-13}$ Genetic markers associated with HBV clearance or hepatitisrelated disease progression may also potentially predict drug treatment efficacy. For example, a single-nucleotide polymorphism (SNP) in STAT4 rs7574865 was shown to be associated with the risk of chronic hepatitis B (CHB), HBV-related liver cirrhosis, and HCC in our previous studies. ${ }^{14,15}$ We further found that rs7574865 was also a potential predictor of treatment response to PegIFN $\alpha$ therapy in hepatitis $\mathrm{B}$ e antigen ( $\mathrm{HBeAg}$ )-positive patients. ${ }^{8,16}$ Similarly, another SNP, rs12614, which is located in the second exon of $C F B$ and identified as a susceptibility locus for $\mathrm{CHB}$, was also significantly associated with the response to PegIFNa therapy in patients with $\mathrm{HBeAg}$-positive CHB. ${ }^{17,18}$

Granulysin (GNLY) is a saposin-like pore-forming protein that acts as a cytotoxic granule and is mainly secreted by cytotoxic $\mathrm{T}$ lymphocytes and natural killer (NK) cells in mammals. It exerts a toxic role with respect to bacteria, fungi, parasites, and tumors, ${ }^{19,20}$ and acts as an anti-virus biomarker, such as in Epstein-Barr virus (EBV) and severe acute respiratory syndrome coronavirus 2 (SARS-CoV-2) infection. $^{21,22}$ Encoded by the $G N L Y$ gene in chromosome 2p11.2, GNLY has two isoforms, unprocessed $15 \mathrm{kDa}$ and the processed $9 \mathrm{kDa}$. The recombinant $9-\mathrm{kDa}$ protein has been demonstrated its broadly cytotoxic and antimicrobial properties, while $15 \mathrm{kDa}$ isoform may function as an immune "alarmin", promoting the maturation and migration of antigen-presenting cells and other immune cells. ${ }^{23}$ It has been reported that GNLY exerts antiviral effects by inhibiting the replication of varicella-zoster virus and triggering apoptosis in infected cells. ${ }^{24}$ However, correlations between GNLY and hepatitis viruses have been limited to association studies, and specific anti-virus mechanistic studies have not been reported. A common missense polymorphism of GNLY, rs11127, was previously reported to be significantly associated with HBV clearance in patients with CHB. ${ }^{25,26}$ However, whether the different genotypes of GNLY rs11127 influence the treatment efficacy of PegIFN $\alpha$ or NUCs remains to be elucidated.

This study first evaluated the predictive performance of GNLY rs11127 in response to CHB therapies in two PegIFN $\alpha$ and two NUCs cohorts. We then compared GNLY expression levels in patients with different GNLY rs11127 genotypes using data from the Genotype-Tissue Expression Project (GTEx). Finally, a polygenic score (PGS) integrating GNLY rs11127 and three other SNPs (STAT4 rs7574865, CFB rs12614, and CD55 rs28371597), ${ }^{8,18}$ which were associated with PegIFNa treatment response, was established to predict the possibility of PegIFN $\alpha$ therapy response.

\section{Patients and Methods}

\section{Patients}

We included a total of $1823 \mathrm{CHB}$ patients from four Phase IV, multicenter, randomized controlled trials, and they were retrospectively analyzed. Patients in two trials (PegIFN $\alpha$ cohort 1, n = 246 and PegIFN $\alpha$ cohort 2, $\mathrm{n}=708$ ) were treated with PegIFN $\alpha-2 a / 2 b$ for at least 48 weeks, and those in the other two trials (NUCs cohort $1, \mathrm{n}=553$, and NUCs cohort $2, \mathrm{n}=316$ ) were treated with NUCs for 104 weeks. Details of patient allocation and specific treatment regimens for each cohort are described in Supplementary Table 1, and the inclusion and exclusion criteria of the patients were previously described. ${ }^{27-30}$ Briefly, eligible patients were aged 18-65 years, with positive $\mathrm{HBsAg}$ for at least 6 months, positive $\mathrm{HBeAg}$, HBV DNA $>1.7 \times 10^{4} \mathrm{IU} / \mathrm{mL}$ $\left(10^{5}\right.$ copies $\left./ \mathrm{mL}\right)$ or $2 \times 10^{4} \mathrm{IU} / \mathrm{mL}$ (PegIFN $\alpha$ cohort 2$)$, and ALT $\geq 2$ (except ALT $\geq 1.3$ in NUCs cohort 2 ) and $<10$ $\times$ upper limits of normal (ULN). We excluded patients who had received antiviral treatment within the prior 6 months or were infected with other chronic liver diseases, including decompensated liver disease and HCC. The study flowchart is provided in Figure 1. 
This study was performed in accordance with the Declaration of Helsinki and was approved by the Ethics Committee of Nanfang Hospital (PegIFN $\alpha$ cohort 1 NCT01086085, PegIFNa cohort 2 - NCT01760122, NUCs cohort 1 - NCT00962533, NUCs cohort 2 NCT01088009). Furthermore, written informed consent was obtained from all participants.

\section{DNA Extraction and Genotyping of Genetic Polymorphisms}

Genomic DNA was extracted from the peripheral blood of patients with CHB using a QIAamp DNA
Blood Mini Kit (Qiagen, Hilden, Germany) according to the manufacturer's instructions. Genotyping of rs11127 was performed using the Sequenom MassARRAY platform (Sequenom, San Diego, CA, USA) according to the instructions at the FudanVARI Center of Genetic Epidemiology, Fudan University. ${ }^{15,16}$ The polymerase chain reaction was conducted using $10 \mathrm{ng}$ of genomic DNA in $5 \mu \mathrm{L}$ volumes. Twenty duplicate test samples were genotyped in a double-blind manner. The genotyping concordance was $100 \%$ for duplicate samples.

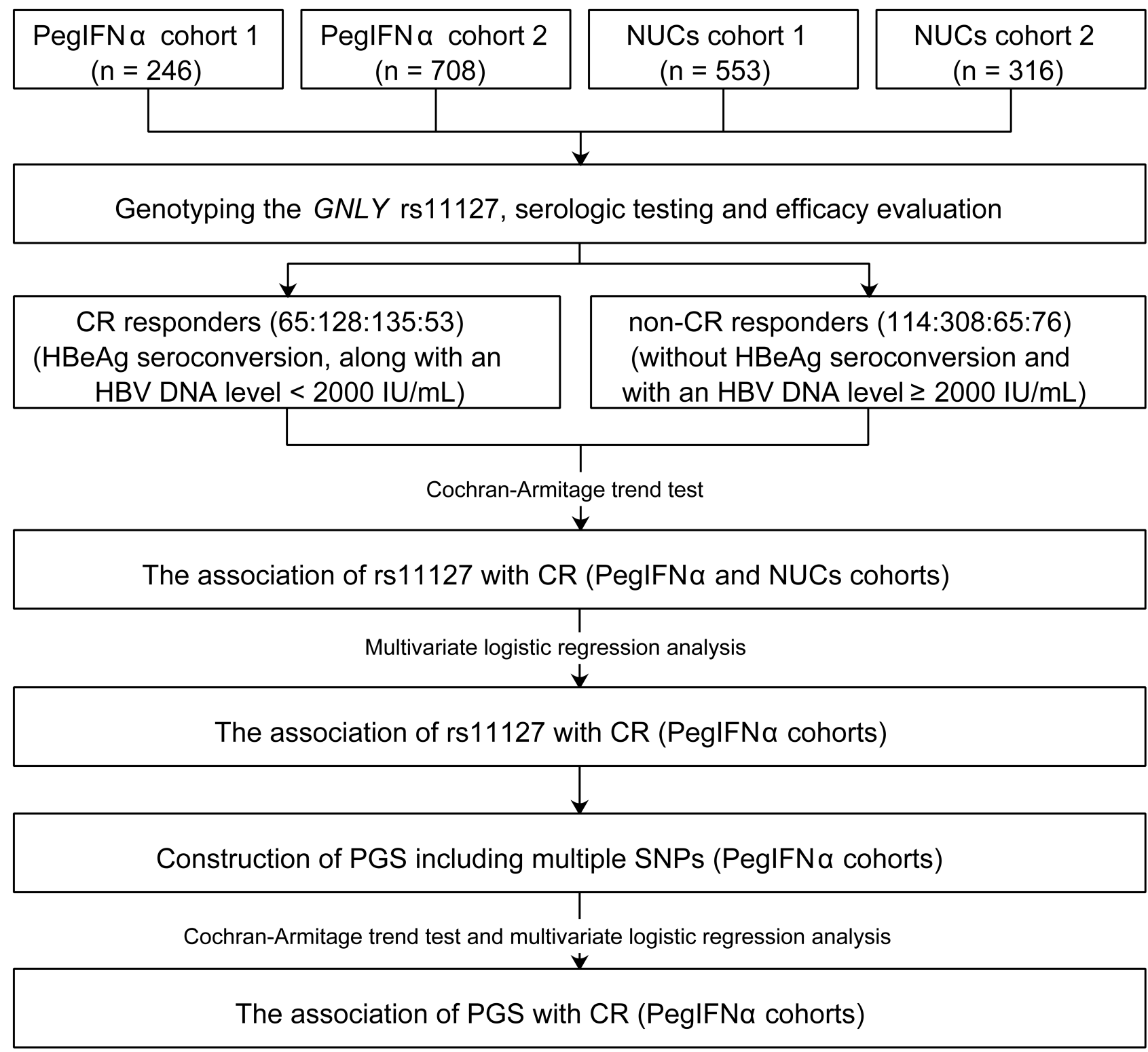

Figure I Flowchart of the study procedure.

Abbreviations: HBV, hepatitis B virus; PegIFNa, pegylated interferon alpha; NUCs, nucleoside analogues; CR, combined response; GNLY, granulysin; HBeAg, hepatitis B e antigen; SNPs, single-nucleotide polymorphisms; PGS, polygenic score. 


\section{Serologic Testing and Efficacy Evaluation}

Clinical laboratory parameters were evaluated every $8-16$ weeks during the trial. HBV DNA levels were detected using the Roche COBAS TaqMan platform (Hoffmann-La Roche Ltd, Basel, Switzerland), with a lower limit of detection (LLOD) of $12 \mathrm{IU} / \mathrm{mL}$ or 69.84 copies $/ \mathrm{mL}$. HBV serological markers were measured using ARCHITECT i2000SR (Abbott Laboratories, Chicago, IL, USA) at the central laboratory of each research group. ${ }^{29}$ Results below the detection limit were substituted by LLOD.

The primary efficacy endpoints were $\mathrm{CR}$ and $\mathrm{HBsAg}$ loss in this study. CR was defined as $\mathrm{HBeAg}$ seroconversion (loss of HBeAg and the presence of $\mathrm{HBeAb}$ ) along with an HBV DNA level $<2000 \mathrm{IU} / \mathrm{mL}$ at week 72 in PegIFN $\alpha$ cohorts or week 104 in NUCs cohorts. HBsAg loss was defined as an HBsAg titer lower than the detection limit (HBsAg $<0.05 \mathrm{IU} / \mathrm{mL}$ ) at the corresponding endpoints. Patients without $\mathrm{HBeAg}$ seroconversion and with an HBV DNA level $\geq 2000 \mathrm{IU} / \mathrm{mL}$ at the corresponding endpoints were classified as non-responders.

\section{Analysis of GNLY Expression Levels in Whole Blood and Liver Tissues with Different rs III27 Alleles}

To further investigate the potential association of the rs11127 genotypes with GNLY expression level, the expression of GNLY in liver tissue or blood and the rs11127 genotype information were downloaded from the GTEx portal website (https://gtexportal.org/home/testyourown). Violin plot was used to visualize GNLY expression levels according to different rs11127 genotypes.

\section{Statistical Analyses}

Continuous variables were presented as mean \pm standard deviation (SD), and categorical variables were presented as counts (percentages). The Hardy-Weinberg equilibrium (HWE) of the rs11127 genotype distribution in CHB patients was assessed using a chi-square test. The association of rs11127 with CR was analyzed using the CochranArmitage trend test. Multiple logistic regression analysis was performed using a backward elimination process, in which only factors with a $P<0.25$ in the univariate logistic regression analysis were included. In addition, the factors with a $P$-value $<0.1$ in the backward selection procedure remained in the model.
To assess the cumulative performance of multiple SNPs, a PGS, as an individual predictor of CHB treatment response, was constructed by counting the number of favorable alleles. Favorable alleles were defined as the alleles associated with a better response to antiviral drugs (allele T in STAT4 rs7574865, C in CFB rs12614, and T in CD55 rs28371597) $)^{8,18}$ for patients with HBV carrying it (homozygous or heterozygous). For each participant, a PGS was calculated as the sum of the favorable alleles $(0-8)$ in the four genes. The association between PGS and CR was assessed using the Cochran-Armitage trend test. Multiple logistic regression analysis was used to evaluate the effect of PGS after adjusting for other baseline variables. In addition, the performance of different regression models for predicting PegIFN $\alpha$ efficacy were compared by receiver operating characteristic curve (ROC) contrast estimation and test. All statistical tests were two-sided. Statistical significance was defined as $P<0.05$. All analyses were performed using SAS 9.3 version 9.1.3 (SAS Institute, Cary, NC).

\section{Results}

\section{Clinical Characteristics}

The demographics, HBV genotypes, and HBsAg levels were generally similar between the PegIFN $\alpha$ and NUCs cohorts, as shown in Table 1. Genotyping of rs11127 failed in 17 patients $(1,12,4$, and 1 , in PegIFN $\alpha$ cohort $1 / 2$ and NUCs cohort $1 / 2$, respectively). Patients were predominantly men and the majority of patients were from Han Chinese ancestry (96.44\% in the PegIFNa cohort and $96.78 \%$ in the NUCs cohort). Only Han Chinese patients were included for further analyses to minimize the influence of genetic heterogeneity. In terms of HBV genotypes, $\mathrm{C}$ was predominant (58.70$61.45 \%)$, followed by B (37.97-38.26\%). HBsAg levels were comparable between the two combined cohorts. Other HBV serological markers at baseline levels, such as HBV DNA and HBeAg, were higher in the PegIFN $\alpha$ than the NUCs cohorts (Table 1). The level of ALT was $4.47 \times$ ULN in the combination of the two PegIFN $\alpha$ cohorts and $3.98 \times \mathrm{ULN}$ in the combination of the two NUCs cohorts, respectively. In addition, the proportions of GNLY rs 11127 (CC, CT, and TT) were very similar in patients treated with PegIFN $\alpha$ and those treated with NUCs $(P=0.635)$, and no obvious correlation with other baseline variables was found in PegIFNa-treated patients and NUCs-treated patients (Supplementary Table 2). 
Table I Characteristics of Patients in PegIFN $\alpha$ Cohorts and NUCs Cohorts

\begin{tabular}{|c|c|c|c|c|c|c|c|}
\hline \multirow[t]{2}{*}{ Clinical Variables } & \multicolumn{3}{|c|}{ PegIFNa Cohorts } & \multicolumn{3}{|c|}{ NUCs Cohorts } & \multirow{2}{*}{$\begin{array}{c}\text { P-value (PegIFN } \alpha \\
\text { Cohort } \\
\text { Combination vs } \\
\text { NUCs Cohort } \\
\text { Combination) }\end{array}$} \\
\hline & $\begin{array}{l}\text { PegIFNa } \\
\text { Cohort I } \\
(n=246)\end{array}$ & $\begin{array}{l}\text { PegIFN } \alpha \\
\text { Cohort } 2 \\
(n=708)\end{array}$ & $\begin{array}{c}\text { PegIFNa } \\
\text { Cohort } \\
\text { Combination } \\
(n=954)\end{array}$ & $\begin{array}{l}\text { NUCs } \\
\text { Cohort I } \\
(n=553)\end{array}$ & $\begin{array}{l}\text { NUCs } \\
\text { Cohort } 2 \\
(n=316)\end{array}$ & $\begin{array}{c}\text { NUCs } \\
\text { Cohort } \\
\text { Combination } \\
(n=869)\end{array}$ & \\
\hline Male gender (\%) & $194(78.90)$ & $512(72.30)$ & $706(74.00)$ & $450(81.40)$ & $239(75.60)$ & 689 (79.29) & 0.008 \\
\hline Age, years; mean (SD) & $29.05(6.79)$ & $29.743(6.70)$ & $29.56(6.73)$ & $30.13(8.96)$ & $31.94(9.40)$ & $30.79(9.16)$ & 0.001 \\
\hline Han ethnicity (\%) & $243(98.80)$ & $677(95.60)$ & $920(96.44)$ & $540(97.70)$ & $301(95.30)$ & $841(96.78)$ & 0.629 \\
\hline HBV genotype (\%) & & & & & & & 0.121 \\
\hline B & $87(35.40)$ & $278(40.40)$ & $365(38.26)$ & $215(38.90)$ & $115(36.40)$ & $330(37.97)$ & \\
\hline C & $157(63.80)$ & $403(58.50)$ & $560(58.70)$ & $335(60.60)$ & $199(63.00)$ & $534(61.45)$ & \\
\hline Others & $2(0.80)$ & $8(1.20)$ & $10(1.04)$ & $3(0.50)$ & $2(0.60)$ & $5(0.59)$ & \\
\hline NA & $0(0.00)$ & $19(2.70)$ & $19(1.99)$ & $0(0.00)$ & $0(0.00)$ & $0(0.00)$ & \\
\hline HBV DNA*, $\log _{10} \mathrm{IU} / \mathrm{mL}$; mean (SD) & $7.69(1.28)$ & $7.93(0.76)$ & $7.87(0.93)$ & $7.71(1.07)$ & $7.80(0.90)$ & $7.74(1.01)$ & 0.006 \\
\hline $\mathrm{HBs} A g^{*}, \log _{10} \mathrm{IU} / \mathrm{mL}$; mean (SD) & $4.01(0.74)$ & $4.28(0.53)$ & $4.21(0.60)$ & $4.20(0.68)$ & $4.18(0.72)$ & $4.19(0.69)$ & 0.579 \\
\hline $\mathrm{HBeAg} * \log _{10} \mathrm{PEIU} / \mathrm{mL} ;$ mean (SD) & $2.42(0.98)$ & $3.04(0.56)$ & $2.88(0.74)$ & $2.74(0.64)$ & $2.72(0.62)$ & $2.73(0.63)$ & $<0.001$ \\
\hline ALT*, ×ULN; mean (SD) & $4.30(3.74)$ & $4.54(2.13)$ & $4.47(2.65)$ & $4.30(3.79)$ & $3.40(2.52)$ & $3.98(3.4 I)$ & $<0.001$ \\
\hline GNLY rs I I I 27 genotype (\%) & & & & & & & 0.635 \\
\hline $\mathrm{CC}$ & $43(17.48)$ & $130(18.36)$ & $173(18.13)$ & $93(16.82)$ & $55(17.41)$ & $148(17.03)$ & \\
\hline $\mathrm{CT}$ & $123(50.00)$ & $284(40.11)$ & $407(42.66)$ & $250(45.21)$ & $140(44.30)$ & $390(44.98)$ & \\
\hline $\mathrm{TT}$ & $76(30.89)$ & $233(32.91)$ & 309 (32.39) & $193(34.90)$ & $106(33.54)$ & $299(34.4 I)$ & \\
\hline NA & $4(1.63)$ & $61(8.62)$ & $65(6.8 I)$ & $17(3.07)$ & $15(4.75)$ & $32(3.68)$ & \\
\hline
\end{tabular}

Notes: *Baseline level. The $P$ values of each baseline variable were calculated by comparing PeglFN $\alpha$ cohort combination and NUCs cohort combination. Abbreviations: PegIFN $\alpha$, pegylated interferon alpha; NUCs, nucleoside analogues; SD, standard deviation; HBV, hepatitis B virus; NA, not available; HBsAg, hepatitis $B$ surface antigen; $\mathrm{HBeAg}$, hepatitis $\mathrm{B}$ e antigen; ALT, alanine aminotransferase; ULN, upper limit of normal; GNLY, granulysin.

\section{Association of GNLY rs I I 27 with CR in the PegIFN $\alpha$ and NUCs Cohorts}

Allele distributions of GNLY rs11127 fulfilled HWE in four cohorts and minor allele frequency (MAF) was $>0.4$ in each cohort. Based on predefined inclusion and exclusion criteria, 179 (responders and non-responders $=65 / 114), 436(128 /$ 308), 199 (135/64) and 129 (53/76) patients remained in PegIFN $\alpha$ cohort $1 / 2$ and NUCs cohort $1 / 2$, respectively.

As shown in Figure 2, 28.57\%, 38.30\%, and $44.83 \%$ of patients with rs11127 TT, CT, and CC genotypes in PegIFNa cohort 1 achieved CR, respectively. In addition, 26.53\%, 26.53\%, and 39.78\% in PegIFNa cohort 2 achieved CR. When combining the two PegIFNa cohorts, we found that the CR rates in patients with the rs $11127 \mathrm{TT}, \mathrm{CT}$, and CC genotypes were $27.09 \%, 30.34 \%$, and $40.98 \%$, respectively. Univariate logistic regression analysis found significant correlations between $G N L Y$ rs11127 and CR both in PegIFNa cohort $2(\mathrm{OR}=1.33, P=0.047)$ and the combined PegIFNa cohort ( $\mathrm{OR}=1.35, P=0.013$, Supplementary Table 3$)$. After adjusting for other baseline variables (the factors with a $P$-value $<0.1$ in the backward selection procedure), significant correlations between $G N L Y$ rs 11127 and CR were also remained in the PegIFN $\alpha$ cohort $2(\mathrm{OR}=1.55, P=0.008)$ and the combined PegIFN $\alpha$ cohort (OR $=1.56, P=0.003$, Figure $2 \mathrm{~A}-\mathrm{C}$ and Table 2). Further, by including clinical variables and SNPs, we constructed a regression model for predicting $\mathrm{CR}$, which was as follow: $\log (\mathrm{P} /(1-\mathrm{P}))=-0.005$ $0.060 *$ Age $+0.685 *$ HBV genotype $-1.143 * \mathrm{HBeAg}+$ $0.902 *$ ALT $+1.137 *$ CFB genotype $+1.503 *$ CD55 genotype $+0.443 * G N L Y$ genotype. However, no statistically significant association between rs11127 and CR rate was found in the two NUCs cohorts (Supplementary Figure 1).

\section{Expression of GNLY in Whole Blood and Liver Tissues Between Different rs I I I27 Alleles}

GTEx data has been representing the largest atlas of the catalog of trait loci and human gene expression. We assessed GNLY expression levels in whole blood and liver tissues with different rs11127 genotypes using the GTEx website to further investigate the effect of the rs11127 genotype on the mRNA expression of GNLY. Different GNLY expression 

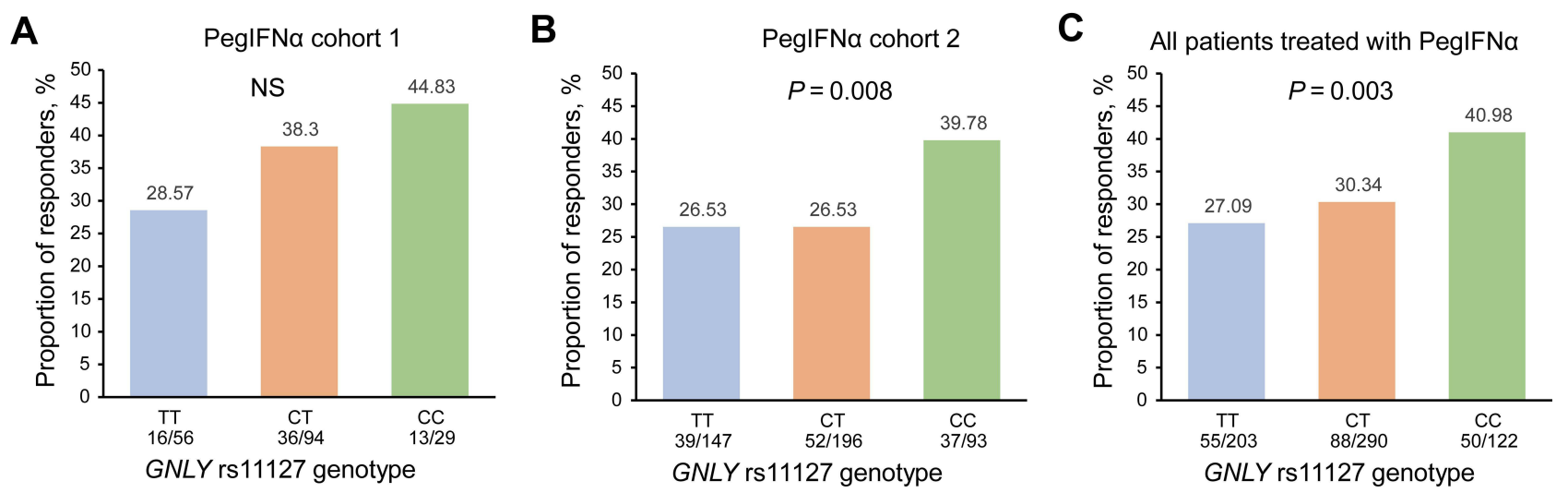

Figure 2 Association of GNLY rs I I 27 with CR in different PegIFN $\alpha$ cohorts. Bar graph showing the association between rs I I 27 genotype and CR in PegIFN $\alpha$ cohort I (A), PegIFN $\alpha$ cohort 2 (B), and all the Chinese HBeAg-positive CHB patients treated with PegIFN $\alpha$ (C). $P$ values were calculated by multiple logistic regression analysis. Abbreviations: NS, no significance; PegIFN $\alpha$, pegylated interferon alpha; GNLY, granulysin.

levels were found among individuals with different genotypes of the genetic variant. As shown in Figure 3, compared with those carrying rs11127 CT or TT, individuals carrying rs11127 CC showed higher GNLY expression levels in whole blood $\left(P=1.700 \times 10^{-27}\right)$ or liver tissues $(P=0.0001)$.

\section{Association of PGS with CR in the PegIFN $\alpha$ Cohorts}

In our previous studies, STAT4 rs7574865, CFB rs12614, and $C D 55$ rs28371597 were identified as reliable predictors of treatment response in patients with $\mathrm{HBeAg}$-positive CHB patients treated with PegIFNa. ${ }^{8,18}$ A PGS was constructed to assess the cumulative effect of the SNPs and GNLY rs11127. For each SNP, homozygous non-favorable alleles, heterozygous, and homozygous for favorable alleles were coded as 0,1 , and 2 , respectively. The PGS was calculated for each participant as the sum of the favorable alleles.

We have found that the $\mathrm{CR}$ rate steadily increased with an increase in PGS (Figure 4A-C). In all patients treated with PegIFN $\alpha$, the CR rates in patients carrying five and six favorable alleles were $48.89 \%$, and $50 \%$, respectively. These CR rates were higher than the proportions of $23.4 \%$, $25.87 \%$, and $32.1 \%$ in patients carrying two, three, and four favorable alleles, respectively. After adjusting for other baseline variables, the association between PGS and CR was significant in PegIFN $\alpha$ cohort $1(\mathrm{OR}=1.87, P=0.004)$, PegIFN $\alpha$ cohort $2(\mathrm{OR}=1.44, P=0.001)$, and the combined PegIFN $\alpha$ cohort $(\mathrm{OR}=1.52, P<0.001$, Table 3$)$.

In addition, we constructed regression models for predicting PegIFN $\alpha$ efficacy by including clinical variables combined with PGS: model 2, or including clinical variables only: model 1 . Through further ROC contrast estimation and testing, the results demonstrated that the performance of the model including clinical variables combined with PGS in predicting PegIFN $\alpha$ efficacy was remarkably improved compared to that of the model including clinical variables only $(P=0.025$, Supplementary Table 4$)$.

\section{Discussion}

It is important to predict the treatment efficiency to target CHB patients, as PEG-IFN is used as a first-line antiviral therapy in adults and the treatment response rate is limited. Previous studies suggest that responseguided therapy or early on-treatment HBV RNA levels could be of great help in the management of patients treated with PegIFNa, ${ }^{29,31}$ while it is unclear which indicators can predict that patients with $\mathrm{CHB}$ could benefit from earlier treatment. Although several SNPs have been proposed, ${ }^{8,32,33}$ the effect of GNLY rs11127 on PegIFN $\alpha$ efficacy has never been reported before. Previously, GNLY rs11127 was regarded as an important factor in HBV clearance in patients infected with HBV in a Korean study. ${ }^{25}$ The present study systematically evaluated the performance of GNLY rs11127 in predicting the response to PegIFN $\alpha$ or NUCs in Chinese $\mathrm{HBeAg}$-positive CHB patients from four multicenter, randomized controlled trials. The missense variant rs 11127 was significantly associated with $\mathrm{CR}$ in PegIFN $\alpha$-treated patients, but not in those treated with NUCs. In addition, we constructed a robust PGS model including STAT4 rs7574865, CFB rs12614, CD55 rs28371597 and GNLY rs11127, which significantly improved the prediction of PegIFN $\alpha$ treatment efficacy. 


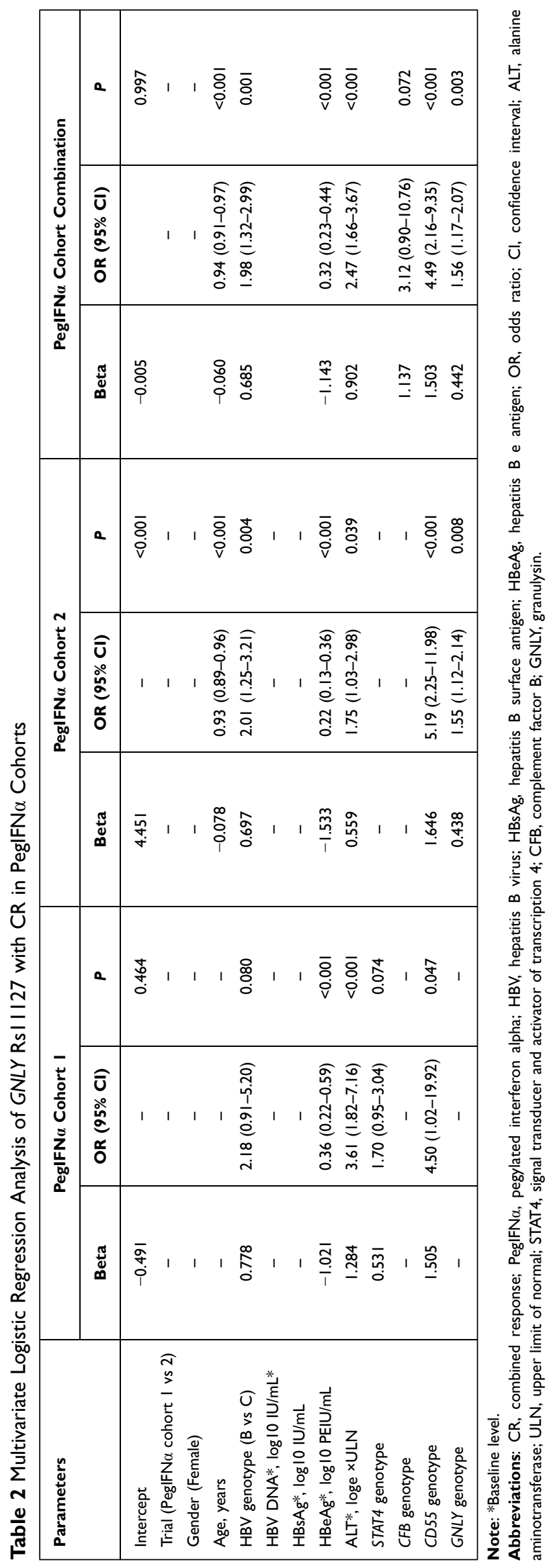

Currently, predictive models of the efficacy for initial treatment with PegIFN were mainly reported on studies of some clinical baseline indicators ${ }^{34}$ or SNPs. ${ }^{32,33,35}$ Therefore, we compared the performance of clinical variables combined with PGS and that of clinical variables only, in predicting PegIFN $\alpha$ efficacy. The results demonstrated that the performance of the model including clinical variables combined with PGS in predicting PegIFN $\alpha$ efficacy was remarkably improved compared to that of the model including clinical variables only. It may also provide a more practical reference for precise treatment strategies for HBeAg-positive CHB patients.

The disparity in the predictive ability of GNLY rs11127 for treatment response to PegIFNa versus NUCs may be due to the different antiviral mechanisms. NUCs exert their antiviral effects by selectively inhibiting the reverse transcriptase domain of virus polymerase to disrupt virus replication. ${ }^{36}$ Unlike NUCs, IFNa has antiviral and immunomodulatory properties, involved in the direct inhibition of viral replication and the enhancement of the host's antiviral immune response. ${ }^{37}$ Besides a "finite" duration of treatment, include the advantages of IFN $\alpha$ treatment included the absence of resistance potential and achievement of a sustained CR due to its potential for immunemediated control of HBV infection. ${ }^{38}$ Furthermore, PegIFN $\alpha$ becomes a crucial component of new regimens and exerts better effects in preventing liver cancer in patients with CHB infection than NUCs. ${ }^{5,39,40}$ However, the treatment response of PegIFN $\alpha$ was only achieved in a small fraction of patients (less than 30\%). Thus, it is necessary to identify new predictive biomarkers.

The current study mainly focused on the potential of GNLY rs11127 in predicting therapeutic response to PegIFN $\alpha$ therapy in patients with HBeAg-positive CHB. GNLY is a critical effector molecule, released mainly by killer lymphocytes, such as CD8+ T cells and NK cells, and exhibits a wide range of antimicrobial activities against bacteria, viruses, and fungi. ${ }^{23}$ The 9-kDa GNLY is broadly cytotoxic and produced by a $15-\mathrm{kDa}$ protein cleaved at its amino- and carboxy termini. The 15-kDa GNLY was reported to function as an immune "alarmin", promoting the maturation and migration of immune cells, such as antigen-presenting cells. This dual function of GNLY indirectly enhances the immune response to infections and enriches its role in the immune system. ${ }^{23}$ It has been proposed that cytotoxic lymphocytes kill bacteriainfected cells by delivering toxic molecules in a two-step process. Perforin generates pores to deliver the lytic 

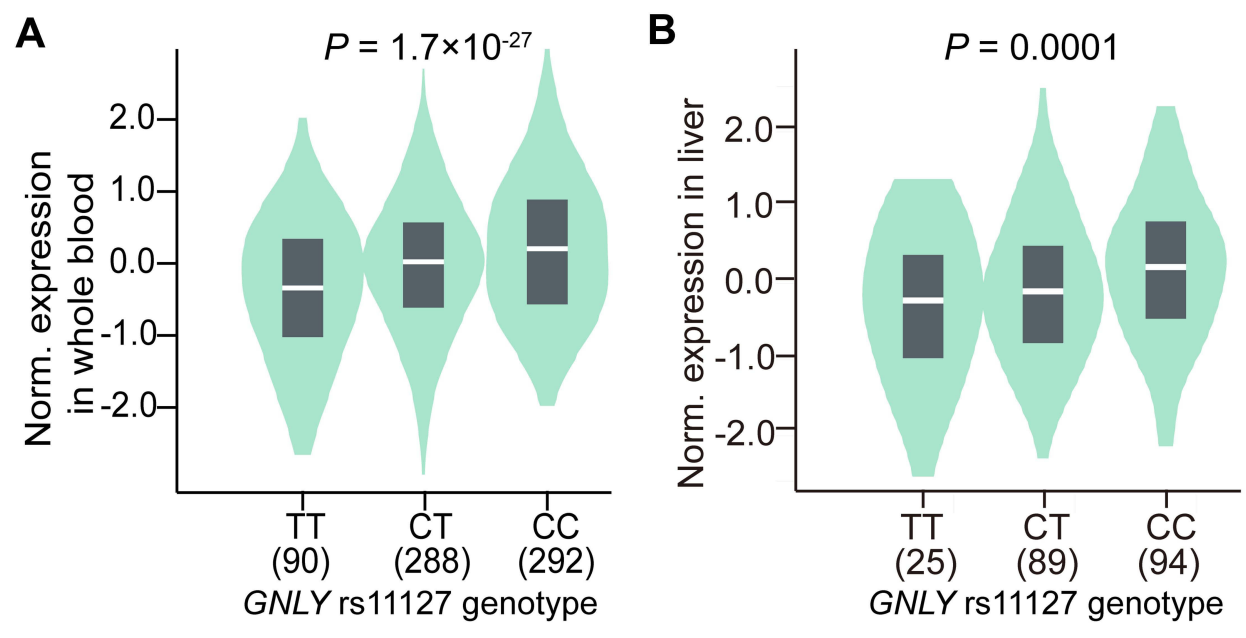

Figure 3 VInPlot showing gene expression of GNLY in whole blood (A) and liver tissues (B) between different rs III 27 genotypes.

Abbreviation: GNLY, granulysin.

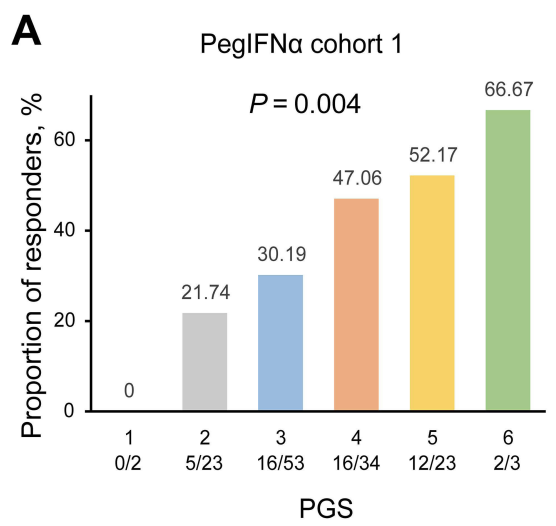

B

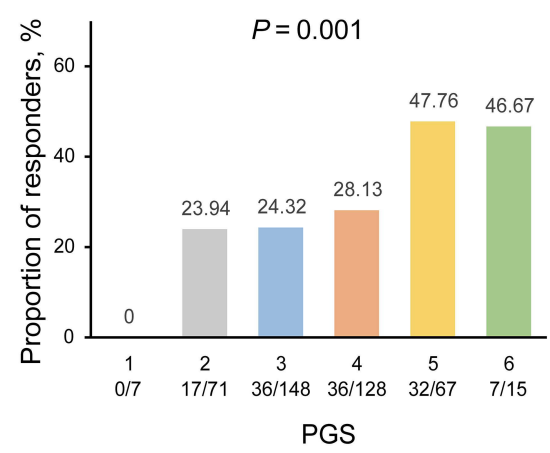

C All patients treated with PegIFNa

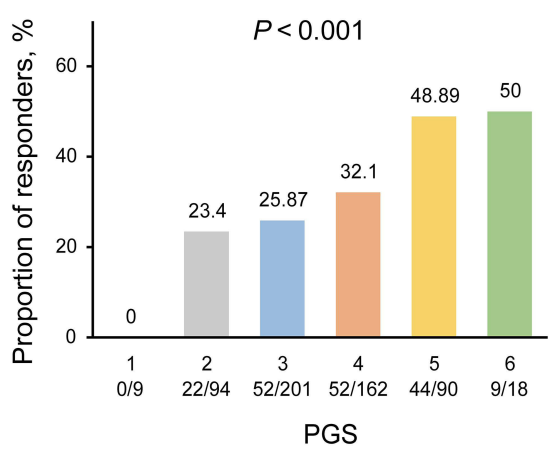

Figure 4 Association of PGS with CR in different PegIFN $\alpha$ cohorts. Bar graph showing the association between PGS and CR in PegIFN $\alpha$ cohort I (A), PegIFN $\alpha$ cohort 2 (B), and all the Chinese $\mathrm{HBeAg}$-positive $\mathrm{CHB}$ patients treated with PegIFN $\alpha(\mathbf{C})$. $P$ values were calculated by multiple logistic regression analysis.

Abbreviations: PegIFN $\alpha$, pegylated interferon alpha; PGS, polygenic score.

granule into the infected cells, and then granulysin delivers granzymes into bacteria to kill diverse bacteria. ${ }^{41}$ However, GNLY is usually reported as a cytolytic effector in viral diseases, such as HBV and SARS-CoV-2 infection, and its antiviral mechanism is still unclear. ${ }^{21}$ The rs11127 SNP, located in the $4^{\text {th }}$ exon of GNLY, changes the amino acid, which may influence the treatment efficacy of PegIFN $\alpha$. Furthermore, data from GTEx revealed that the GNLY mRNA expression levels varied depending on the rs11127 genotypes, which may be caused by the change in mRNA stability or potential linkage disequilibrium between the SNP and some functional sites in the regulatory region.

A previous study on the association of GNLY genetic polymorphisms with CHB infection in Korea showed that the $\mathrm{T}$ allele-containing genotype of rs 11127 was associated with HBV clearance. ${ }^{25}$ However, in this study, we found that the $\mathrm{C}$ allele of rs 11127 was the favorable allele with a higher CR in patients with CHB treated with PegIFN $\alpha$. Our results were consistent with those of a Chinese study, which showed significantly reduced susceptibility to HBV infection in men carrying the rs11127 TC genotype and women carrying the rs11127 CC genotype. ${ }^{26}$ However, the underlying mechanism of these discrepant results remains unclear. A possible explanation is the different ethnic and hereditary backgrounds of the Korean and Chinese patients.

With the continuous decrease in the cost of genotyping chips, PGS has great potential for the precise prediction of diseases or treatment efficacy. Gellert-Kristensen found a genetic risk score including three common variants conferred 
Table 3 Multivariate Logistic Regression Analysis of PGS with CR in PegIFN $\alpha$ Cohorts

\begin{tabular}{|c|c|c|c|c|c|c|}
\hline \multirow[t]{2}{*}{ Parameters } & \multicolumn{2}{|c|}{ PegIFN $\alpha$ Cohort I } & \multicolumn{2}{|c|}{ PegIFN $\alpha$ Cohort 2} & \multicolumn{2}{|c|}{ PegIFN $\alpha$ Cohort Combination } \\
\hline & OR $(95 \% \mathrm{Cl})$ & $\boldsymbol{P}$ & OR $(95 \% \mathrm{Cl})$ & $\boldsymbol{P}$ & OR $(95 \% \mathrm{Cl})$ & $\mathbf{P}$ \\
\hline Trial (PegIFN $\alpha$ cohort I vs 2 ) & - & - & - & - & $0.79(0.48-1.32)$ & 0.376 \\
\hline Gender (Female) & $0.87(0.31-2.45)$ & 0.788 & $1.38(0.82-2.33)$ & 0.223 & $1.29(0.82-2.05)$ & 0.277 \\
\hline Age, years & $0.96(0.90-1.03)$ & 0.228 & $0.93(0.90-0.97)$ & $<0.001$ & $0.94(0.91-0.97)$ & $<0.001$ \\
\hline HBV genotype (B vs $C$ ) & $2.58(1.00-6.68)$ & 0.050 & $1.99(1.23-3.21)$ & 0.005 & $2.06(1.35-3.14)$ & $<0.001$ \\
\hline HBV DNA*, $\log _{10} \mathrm{IU} / \mathrm{mL}$ & $1.33(0.75-2.38)$ & 0.328 & $0.97(0.63-1.47)$ & 0.869 & $1.16(0.84-1.6)$ & 0.377 \\
\hline $\mathrm{HBsAg} * \log _{10} \mathrm{IU} / \mathrm{mL}$ & $0.57(0.24-1.33)$ & 0.192 & $0.97(0.53-1.76)$ & 0.913 & $0.79(0.49-1.26)$ & 0.318 \\
\hline $\mathrm{HBeAg} *, \log _{10} \mathrm{PEIU} / \mathrm{mL}$ & $0.34(0.17-0.66)$ & 0.001 & $0.25(0.14-0.43)$ & $<0.001$ & $0.31(0.21-0.46)$ & $<0.001$ \\
\hline $\mathrm{ALT}^{*}, \log _{\mathrm{e}} \times \mathrm{ULN}$ & $3.64(1.77-7.48)$ & $<0.001$ & $1.86(1.09-3.17)$ & 0.023 & $2.53(1.69-3.79)$ & $<0.001$ \\
\hline PGS & $1.87(1.23-2.85)$ & 0.004 & $1.44(1.15-1.80)$ & 0.001 & $1.52(1.25-1.85)$ & $<0.001$ \\
\hline
\end{tabular}

Note: *Baseline level.

Abbreviations: $C R$, combined response; PegIFN $\alpha$, pegylated interferon alpha; HBV, hepatitis B virus; HBsAg, hepatitis B surface antigen; HBeAg, hepatitis B e antigen; OR, odds ratio; $\mathrm{Cl}$, confidence interval; $\mathrm{ALT}$, alanine aminotransferase; ULN, upper limit of normal; PGS, polygenic score.

up to a 12-fold higher risk of cirrhosis, and a 29-fold higher risk of HCC in patients with fatty liver disease. ${ }^{42}$ Thus, this study, we constructed a PGS model to evaluate the cumulative effect of GNLY rs11127, STAT4 rs7574865, CFB rs12614, and $C D 55$ rs28371597, all of which were previously identified as predictors of treatment response to PegIFN $\alpha$ therapy. We found that the CR rate increased steadily with an increase in PGS in the PegIFN $\alpha$ cohorts. The CR rate in patients with a PGS of six was approximately 1-fold higher than that in patients with a PGS of 2 or 3 . No individuals with a PGS equal to one achieved a treatment response to PegIFN $\alpha$ therapy, and nearly half of the patients could benefit from PegIFN $\alpha$ therapy if $P G S \geq 5$. The PGS has implications for the clinical management and selection of PegIFNa therapy in HBeAg-positive CHB patients. We expect PGS to comprise more IFN $\alpha$ treatment response-related SNPs, which would be more effective in predicting the treatment response to PegIFN $\alpha$.

Compared with NUCs, PegIFNa was reported to have a greater chance of achieving $\mathrm{HBeAg}$ seroconversion and HBsAg clearance, which are associated with improved clinical outcomes. However, no significant association of rs11127 with HBsAg loss was found in the PegIFNa cohorts (Supplementary Figure 2) because of the limited sample size which attenuated the statistical power. Therefore, further studies with larger sample sizes are needed to validate the association between rs11127 and HBsAg loss in the future.

\section{Conclusions}

GNLY rs11127 was significantly associated with treatment response to PegIFN $\alpha$ therapy in Chinese patients with HBeAg-positive CHB. A PGS comprising GNLY, STAT4, $C F B$, and $C D 55$ variants could effectively predict the CR rate in PegIFN $\alpha$-treated CHB patients, which may have implications for clinical management and individualized treatment guidance.

\section{Abbreviations}

ALT, alanine aminotransferase; $\mathrm{CHB}$, chronic hepatitis $\mathrm{B}$; CR, combined response; GNLY, Granulysin; GTEx, Genotype-Tissue Expression Project; $\mathrm{HBeAg}$, hepatitis B e antigen; HBsAg, hepatitis $\mathrm{B}$ s antigen; HBV, hepatitis $\mathrm{B}$ virus; HCC, hepatocellular carcinoma; HCV, hepatitis $\mathrm{C}$ virus; HWE, Hardy-Weinberg equilibrium; MAF, minor allele frequency; NK, natural killer; NUCs, nucleos(t)ide analogs; PegIFNa, pegylated-interferon-alpha; PGS, polygenic score; ROC, receiver operating characteristic curve; SARS-CoV-2, severe acute respiratory syndrome coronavirus 2; SD, standard deviation; SNPs, single-nucleotide polymorphisms; ULN, upper limit of normal.

\section{Data Sharing Statement}

All data generated or analysed during this study are included in this published article and its additional files. The public data during the current study are available in the GTEx (https://gtexportal.org/home/testyourown).

\section{Ethics Approval and Informed Consent}

This study was approved by the Ethics Committee of Nanfang Hospital. Written informed consent was obtained from all patients or their family members. The data from GTEx are shared and available to the public, and there are no ethical concerns. The study was performed and reported in accordance with the Helsinki Declaration. 


\section{Acknowledgments}

The data used for the analyses described in this manuscript were obtained from the GTEx Portal. We thank Editage for polishing this manuscript.

\section{Funding}

This study was supported by the Local Innovative and Research Teams Project of Guangdong Pearl River Talents Program (No. 2017BT01S131), the National Science and Technology Major Project (No. 2017ZX10202202 and 2018ZX10301202), the General Programs from the National Natural Science Foundation of China (No. 81472618, 81670535, 81802828 and 81802833), the General Program from the Natural Science Foundation of Guangdong Province (No. 2019A1515011423), the Key-Area Research and Development Program of Guangdong Province (No. 2019B020227004), the Innovative Research Team Project of Guangxi Province (No. 2017GXNSFGA198002), the Dean Fund of Nanfang Hospital, Southern Medical University (No. 2018Z005), the Grant for Recruited Talents to Start Scientific Research from Nanfang Hospital, and the Outstanding Youth Development Scheme of Nanfang Hospital, Southern Medical University (No. 2017J001).

\section{Disclosure}

Dr Jinlin Hou reports personal fees from AbbVie, Arbutus, Gilead Sciences, and Roche; grants, personal fees from Bristol Myers Squibb and Johnson\&Johnson, outside the submitted work. The authors declare that they have no other competing interests.

\section{References}

1. Razavi-Shearer D, Gamkrelidze I, Nguyen MH. Global prevalence, treatment, and prevention of hepatitis B virus infection in 2016: a modelling study. Lancet Gastroenterol Hepatol. 2018;3 (6):383-403. doi:10.1016/S2468-1253(18)30056-6

2. Seto WK, Lo YR, Pawlotsky JM, Yuen MF. Chronic hepatitis B virus infection. Lancet. 2018;392(10161):2313-2324. doi:10.1016/S01406736(18)31865-8

3. Tang LSY, Covert E, Wilson E, Kottilil S. Chronic hepatitis B infection: a review. JAMA. 2018;319(17):1802-1813. doi:10.1001/ jama.2018.3795

4. Marcellin P, Bonino F, Lau GK, et al. Sustained response of hepatitis $\mathrm{B}$ e antigen-negative patients 3 years after treatment with peginterferon alpha-2a. Gastroenterology. 2009;136(7):2169-2179 e1-e4. doi:10.1053/j.gastro.2009.03.006

5. Lin SM, Yu ML, Lee CM, et al. Interferon therapy in HBeAg positive chronic hepatitis reduces progression to cirrhosis and hepatocellular carcinoma. $J$ Hepatol. 2007;46(1):45-52. doi:10.1016/j. jhep.2006.08.021

6. Terrault NA, Lok ASF, McMahon BJ, et al. Update on prevention, diagnosis, and treatment of chronic hepatitis B: AASLD 2018 hepatitis B guidance. Hepatology. 2018;67(4):1560-1599.
7. Hou FQ, Song LW, Yuan Q, et al. Quantitative hepatitis B core antibody level is a new predictor for treatment response in HBeAg-positive chronic hepatitis B patients receiving peginterferon. Theranostics. 2015;5(3):218-226. doi:10.7150/thno.10636

8. Chen H, Sun J, Zhou B, et al. Variants in STAT4 associated with cure of chronic HBV infection in HBeAg-positive patients treated with pegylated interferon-alpha. Clin Gastroenterol Hepatol. 2020;18 (1):196-204 e8. doi:10.1016/j.cgh.2019.04.044

9. Trepo C, Chan HL, Lok A. Hepatitis B virus infection. Lancet. 2014;384(9959):2053-2063. doi:10.1016/S0140-6736(14)60220-8

10. Xun Z, Lin J, Yu Q, et al. Taurocholic acid inhibits the response to interferon- $\alpha$ therapy in patients with HBeAg-positive chronic hepatitis B by impairing CD8+ T and NK cell function. Cell Mol Immunol. 2021;18(2):461-471. doi:10.1038/s41423-020-00601-8

11. Ge D, Fellay J, Thompson AJ, et al. Genetic variation in IL28B predicts hepatitis $\mathrm{C}$ treatment-induced viral clearance. Nature. 2009;461(7262):399-401. doi:10.1038/nature08309

12. Thomas DL, Thio CL, Martin MP, et al. Genetic variation in IL28B and spontaneous clearance of hepatitis C virus. Nature. 2009;461 (7265):798-801. doi:10.1038/nature08463

13. Prokunina-Olsson L, Muchmore B, Tang W, et al. A variant upstream of IFNL3 (IL28B) creating a new interferon gene IFNL4 is associated with impaired clearance of hepatitis C virus. Nat Genet. 2013;45 (2):164-171. doi:10.1038/ng.2521

14. Jiang DK, Sun J, Cao G, et al. Genetic variants in STAT4 and HLA-DQ genes confer risk of hepatitis B virus-related hepatocellular carcinoma. Nat Genet. 2013;45(1):72-75. doi:10.1038/ng.2483

15. Jiang DK, Ma XP, Wu X, et al. Genetic variations in STAT4,C2, HLA-DRB1 and HLA-DQ associated with risk of hepatitis B virus-related liver cirrhosis. Sci Rep. 2015;5:16278. doi:10.1038/ srep 16278

16. Jiang DK, Wu X, Qian J, et al. Genetic variation in STAT4 predicts response to interferon-alpha therapy for hepatitis B e antigen-positive chronic hepatitis B. Hepatology. 2016;63(4):1102-1111. doi:10.1002/ hep. 28423

17. Jiang DK, Ma XP, Yu H, et al. Genetic variants in five novel loci including $\mathrm{CFB}$ and $\mathrm{CD} 40$ predispose to chronic hepatitis B. Hepatology. 2015;62(1):118-128. doi:10.1002/hep.27794

18. Chen H, Sun J, Zhou B, et al. A missense variant in complement factor $\mathrm{B}$ (CFB) is a potential predictor of 24-week off-treatment response to PegIFNalpha therapy in Chinese HBeAg-positive chronic hepatitis B patients. Aliment Pharmacol Ther. 2020;51(4):469-478. doi:10.1111/apt.15624

19. Junqueira C, Barbosa CRR, Costa PAC, et al. Cytotoxic CD8(+) $\mathrm{T}$ cells recognize and kill Plasmodium vivax-infected reticulocytes. Nat Med. 2018;24(9):1330-1336. doi:10.1038/s41591-018-0117-4

20. Lu CC, Wu TS, Hsu YJ, et al. NK cells kill mycobacteria directly by releasing perforin and granulysin. $J$ Leukoc Biol. 2014;96 (6):1119-1129. doi:10.1189/jlb.4A0713-363RR

21. Zhang JY, Wang XM, Xing X, et al. Single-cell landscape of immunological responses in patients with COVID-19. Nat Immunol. 2020;21(9):1107-1118. doi:10.1038/s41590-020-0762-x

22. Sun Q, Burton RL, Lucas KG. Cytokine production and cytolytic mechanism of CD4(+) cytotoxic T lymphocytes in ex vivo expanded therapeutic Epstein-Barr virus-specific T-cell cultures. Blood. 2002;99(9):3302-3309. doi:10.1182/blood.V99.9.3302

23. Sparrow E, Bodman-Smith MD. Granulysin: the attractive side of a natural born killer. Immunol Lett. 2020;217:126-132. doi:10.1016/j. imlet.2019.11.005

24. Hata A, Zerboni L, Sommer M, et al. Granulysin blocks replication of varicella-zoster virus and triggers apoptosis of infected cells. Viral Immunol. 2001;14(2):125-133. doi:10.1089/088282401750234501

25. Park GH, Kim KY, Cheong JY, Cho SW, Kwack K. Association of GNLY genetic polymorphisms with chronic liver disease in a Korean population. DNA Cell Biol. 2012;31(9):1492-1498. doi:10.1089/ dna.2012.1709 
26. Hou SH, Hu J, Zhang Y, Li QL, Guo JJ. Effects of interaction between genetic variants in human leukocyte antigen DQ and granulysin genes in Chinese Han subjects infected with hepatitis B virus. Microbiol Immunol. 2015;59(4):209-218. doi:10.1111/1348-0421.12239

27. Sun J, Xie Q, Tan D, et al. The 104-week efficacy and safety of telbivudine-based optimization strategy in chronic hepatitis B patients: a randomized, controlled study. Hepatology. 2014;59 (4):1283-1292. doi:10.1002/hep.26885

28. Liang X, Cheng J, Sun Y, et al. Randomized, three-arm study to optimize lamivudine efficacy in hepatitis B e antigen-positive chronic hepatitis B patients. J Gastroenterol Hepatol. 2015;30(4):748-755. doi:10.1111/jgh.12835

29. Sun J, Ma H, Xie Q, et al. Response-guided peginterferon therapy in patients with $\mathrm{HBeAg}$-positive chronic hepatitis $\mathrm{B}$ : a randomized controlled study. J Hepatol. 2016;65(4):674-682. doi:10.1016/j. jhep.2016.05.024

30. Hou FQ, Yin YL, Zeng LY, et al. Clinical effect and safety of pegylated interferon-alpha-2b injection (Y shape, $40 \mathrm{kD}$ ) in treatment of HBeAg-positive chronic hepatitis B patients. Zhonghua Gan Zang Bing Za Zhi. 2017;25(8):589-596.

31. van Campenhout MJH, van Bommel F, Pfefferkorn M, et al. Serum hepatitis B virus RNA predicts response to peginterferon treatment in HBeAg-positive chronic hepatitis B. J Viral Hepat. 2020;27 (6):610-619. doi:10.1111/jvh.13272

32. Enomoto H, Aizawa N, Hasegawa K, et al. Possible relevance of PNPLA3 and TLL1 gene polymorphisms to the efficacy of PEG-IFN therapy for HBV-infected patients. Int J Mol Sci. 2020;21(9):3089. doi:10.3390/ijms21093089

33. Brouwer WP, Chan HLY, Lampertico P, et al. Genome-wide association study identifies genetic variants associated with early and sustained response to (pegylated) interferon in chronic hepatitis B patients: the GIANT-B study. Clin Infect Dis. 2019;69 (11):1969-1979. doi:10.1093/cid/ciz084

34. Chan HLY, Messinger D, Papatheodoridis GV, et al. A baseline tool for predicting response to peginterferon alfa-2a in $\mathrm{HBeAg}$-positive patients with chronic hepatitis B. Aliment Pharmacol Ther. 2018;48 (5):547-555. doi:10.1111/apt.14862
35. Xie DY, Wang SM, Yang JM, et al. IFIT1 polymorphisms predict interferon-alpha treatment efficiency for hepatitis B virus infection. World J Gastroenterol. 2016;22(44):9813-9821. doi:10.3748/wjg. v22.i44.9813

36. Pei Y, Wang C, Yan SF, Liu LG. Past, current, and future developments of therapeutic agents for treatment of chronic hepatitis B virus infection. J Med Chem. 2017;60(15):6461-6479. doi:10.1021/acs. jmedchem.6b01442

37. Lampertico P, Vigano M, Colombo $\mathrm{M}$. Why do I treat HBeAg-negative chronic hepatitis $B$ patients with pegylated interferon? Liver Int. 2013;33(Suppl 1):157-163. doi:10.1111/ liv. 12064

38. Kao JH. HBeAg-positive chronic hepatitis B: why do I treat my patients with pegylated interferon? Liver Int. 2014;34(Suppl 1):112-119. doi:10.1111/liv.12400

39. Bazinet M, Pantea V, Placinta G, et al. Safety and efficacy of 48 weeks REP 2139 or REP 2165, tenofovir disoproxil, and pegylated interferon Alfa-2a in patients with chronic HBV infection naive to nucleos(t)ide therapy. Gastroenterology. 2020;158(8):2180-2194. doi:10.1053/j.gastro.2020.02.058

40. Liang KH, Hsu CW, Chang ML, Chen YC, Lai MW, Yeh CT. Peginterferon is superior to nucleos $(\mathrm{t})$ ide analogues for prevention of hepatocellular carcinoma in chronic hepatitis B. J Infect Dis. 2016;213(6):966-974. doi:10.1093/infdis/jiv547

41. Walch M, Dotiwala F, Mulik S, et al. Cytotoxic cells kill intracellular bacteria through granulysin-mediated delivery of granzymes. Cell. 2014;157(6):1309-1323. doi:10.1016/j.cell.2014.03.062

42. Gellert-Kristensen H, Richardson TG, Davey Smith G, Nordestgaard BG, Tybjaerg-Hansen A, Stender S. Combined effect of PNPLA3, TM6SF2, and HSD17B13 variants on risk of cirrhosis and hepatocellular carcinoma in the general population. Hepatology. 2020;72(3):845-856. doi:10.1002/hep.31238
Pharmacogenomics and Personalized Medicine

\section{Publish your work in this journal}

Pharmacogenomics and Personalized Medicine is an international, peer-reviewed, open access journal characterizing the influence of genotype on pharmacology leading to the development of personalized treatment programs and individualized drug selection for improved safety, efficacy and sustainability. This journal is indexed on the American Chemical Society's Chemical Abstracts Service (CAS). The manuscript management system is completely online and includes a very quick and fair peer-review system, which is all easy to use. Visit http://www.dovepress.com/testimonials.php to read real quotes from published authors. 